Research Paper

\title{
Inhibition of Autophagy with Chloroquine Enhanced Sinoporphyrin Sodium Mediated Photodynamic Therapy-induced Apoptosis in Human Colorectal Cancer Cells
}

Bing Zhu ${ }^{*}$, Shanxin Li $1^{*}$, Lei Yu ${ }^{3 *}$, Wei Hu ${ }^{4}$, Dandan Sheng 2, Jing Hou 5, Naping Zhao 1, Xiaojuan Hou 2, Yechen $\mathrm{Wu}^{2}$, Zhipeng Han ${ }^{2}$, Lixin Wei ${ }^{{ }^{凶}}$, Li Zhang ${ }^{4,1^{凶}}$

1. Department of Pharmacy, Changhai Hospital, The Second Military Medical University, Shanghai, China

2. Tumor Immunology and Gene Therapy Center, Eastern Hepatobiliary Surgery Hospital, The Second Military Medical University, Shanghai, China

3. Department of Information, Changzheng Hospital, The Second Military Medical University, Shanghai, China

4. Department of Pharmacy, The Second Hospital of Anhui Medical University, Hefei, China

5. GCP Office, Xinhua Hospital Affiliated to Shanghai Jiao Tong University School of Medicine, Shanghai, China

* These authors contributed equally to this work.

$\triangle$ Corresponding author: Li Zhang, E-mail: chyyzhl@163.com; Lixin Wei, E-mail: weilixin_smmu@163.com.

(c) Ivyspring International Publisher. This is an open access article distributed under the terms of the Creative Commons Attribution (CC BY-NC) license (https://creativecommons.org/licenses/by-nc/4.0/). See http://ivyspring.com/terms for full terms and conditions.

Received: 2018.05.08; Accepted: 2018.09.28; Published: 2019.01.01

\begin{abstract}
To evaluate the antitumor effect of sinoporphyrin sodium mediated photodynamic therapy (DVDMS-PDT) against human colorectal cancer (CRC) and to investigate the role of autophagy in its effect.

Shrunken cells, condensed nuclei and increased levels of cleaved caspase- 3 and Bax were observed in DVDMS-PDT treated HCT116 cells, reminiscent of apoptosis. DVDMS-PDT showed better antitumor efficiency in HCT1 16 cells than Photofrin mediated photodynamic therapy (PF-PDT) both in vitro and in vivo. And DVDMS-PDT caused autophagic characteristics: double membrane autophagosome structures and changes in autophagy-related protein expression (ATG7, P62, Bcl-2 and LC3-II). In addition, inhibition of autophagy by chloroquine (CQ) promoted apoptosis, suggesting a possible protective role of autophagy in DVDMS-PDT-treated HCT116 cells, which was proved by flow cytometry and western blotting. The results of xenograft mouse model showed markedly increased apoptosis and significantly reduced tumor size in DVDMS-PDT treated group than Control, and DVDMS-PDT exhibited better antitumor efficiency than PF-PDT. Further, no visible tumor was observed in the CQ+DVDMS-PDT group at the end of the xenograft mouse experiment, which confirmed the hypothesis that autophagy was protective to DVDMS-PDT treated HCT116 cells.

Our findings suggest that DVDMS is a promising photosensitizer and the combined use of autophagy inhibitor can remarkably enhance the DVDMS-PDT mediated anti-cancer efficiency in HCT116 cells both in vitro and in vivo.
\end{abstract}

Key words: sinoporphyin sodium, photodynamic therapy, colorectal cancer, apoptosis, autophagy, chloroquine

\section{Introduction}

Colorectal cancer (CRC) is one of the most common cancers globally. In the United States, CRC incidence and mortality rates have been declining for several decades; however, more research is needed to elucidate the increasing burden of CRC in young adults and advanced treatments for tumor subtypes with low response rates to current therapies. Nowadays, CRC remains a major cause of morbidity 
and mortality and a substantial economic burden [1-3]. For patients with unresectable metastatic CRC lesions, the 5-year survival rate drops to only 5\% [4]. In addition to surgery, radiation therapy and chemotherapy are therapeutic strategies for advanced metastatic CRC patients; however, severe side effects are frequently observed. Photodynamic therapy (PDT) is a promising noninvasive therapeutic approach for malignant and premalignant tumors and other diseases, including advanced CRC $[5,6]$. This technique involves the use of photosensitizer (PS) and activation of cellular substrates or endogenous molecular oxygen using a specific wavelength of laser light, producing reactive oxygen species (ROS), further inducing subcellular damages at the sites of PS accumulation, which eventually leads to cell death either by apoptosis and/or necrosis [7, 8].

PS is a critical component of PDT. Ideal PS should possess characteristics such as high chemical stability, good water-solubility, high phototoxicity, optimal wavelength of light source (600-850 nm), low dark toxicity, preferential accumulation in the tumor tissues, and rapid clearance in patients [5, 9]. Researchers have found the high-efficiency fraction of Photofrin (PF), named as sinoporphyin sodium (DVDMS). Early studies suggested that DVDMS possesses excellent properties and great potential for clinical photodynamic therapy [10-12], which has been approved by the Chinese State Food and Drug Administration (SFDA) for conducting phase I clinical trial in patients with advanced oesophageal cancer.

Autophagy is a lysosomal degradation process of cellular organelles and protein recycling under stressful conditions, and has been shown to play important roles in cell survival, differentiation, and development. Recent studies have shown that autophagy is involved in cancer development and progression, and is activated by anti-cancer therapies [13]. Some studies suggested that PDT could induce both apoptosis and autophagy, and autophagy might play controversial roles depending on the cell types and photosensitizers [8, 14, 15]. Andrzejak et al. reported that silencing of the autophagy gene ATG7 increased phototoxicity to mouse leukemia L1210 and 1c1c7 cells [14, 16, 17]. However, in human breast cancer MCF-7 cells, silencing of ATG7 enhanced resistance to PDT [15]. Therefore, further studies are necessary to understand the roles of autophagy in DVDMS-PDT treatment in CRC.

Until now, the functions of DVDMS in PDT remain poorly understood. To our knowledge, DVDMS-PDT has not been investigated in CRC models, and there are no reports on its roles in simultaneously inducing apoptosis and autophagy. In present study, we found DVDMS-PDT induced apoptosis and autophagy in HCT116 human colorectal cancer cells, and DVDMS-PDT showed better anti-cancer effects than PF-PDT both in vitro and in vivo. Furthermore, inhibition of autophagy by $C Q$, a lysosomotropic autophagy inhibitor, increased the cytotoxic effects of DVDMS-PDT on HCT116 cells both in vitro and in vivo. These results suggested that autophagy inhibition can potentiate DVDMS-PDT in colorectal cancer cell, and may provide new choices for the clinical therapy of CRC.

\section{Materials and methods}

\section{Cell culture}

HCT116 cell line was obtained from Cell Resource Center, Chinese Academy of Medical Science, Shanghai, China. Cells were cultured in RIPM 1640 medium (GIBCO, Invitrogen, Carlsbad, CA, USA) supplemented with $10 \%$ fetal bovine serum (FBS, GIBCO) and 1\% penicillin-streptomycin mixture solution (Thermo Fisher Scientific, USA) in a humidified incubator under $95 \%$ air and $5 \% \mathrm{CO}_{2}$ at $37^{\circ} \mathrm{C}$.

\section{Chemicals}

DVDMS (purity: 98.5\%) was provided by Qinglong Hi-tech Co., Ltd (Jiangxi, China). DVDMS stock solution was prepared in physiological saline at $1.00 \mathrm{mg} / \mathrm{mL}$, sterilized, aliquoted and maintained in the dark at $-20^{\circ} \mathrm{C}$ for less than 30 days.

Chloroquine phosphate salt was purchased from Sigma Aldrich (Germany), DAPI was from Beyotime (Shanghai, China) and bovine serum albumin (BSA) was from Bioscience (Shanghai, China). Paraformaldehyde (PFA), glutaraldehyde (GA), Triton X-100 and crystal violet were purchased from Bio-Light Biotech (Shanghai, China).

\section{Detection of DVDMS uptake}

HCT116 cells were incubated with DVDMS (1 $\mu \mathrm{g} / \mathrm{mL}$ ) at $37^{\circ} \mathrm{C}$ for different time intervals. For determining the intracellular DVDMS quantity, cells were collected at time points $(0,0.5,1,2,3,4,6,8,10 \mathrm{~h})$ and detected with flow cytometry (FACSCalibur, BD, USA). The mean fluorescence intensity (MFI) of DVDMS and the percentage of DVDMS uptake cells were recorded (excitation wavelength: $630 \mathrm{~nm}$, emission wavelength: 790nm).

\section{Photodynamic treatment}

The semiconductor laser device (excitation wavelength: $630 \mathrm{~nm}$; manufacturer: Xingda photoelectric medical instrument Co., Ltd., Guangxi, China) was used as a source for evocation of the photodynamic effects. For in vitro experiments, the laser was used with a power intensity of 19.1 
$\mathrm{mW} / \mathrm{cm}^{2}$ (power: $1500 \mathrm{~mW}$, diameter: $10 \mathrm{~cm}$ ) and an irradiation time of $265 \mathrm{~s}$ to $530 \mathrm{~s}$ to achieve the final light dose ranging from 5 to $10 \mathrm{~J} / \mathrm{cm}^{2}$. For in vivo experiment, the laser was used with a power intensity of $318.5 \mathrm{~mW} / \mathrm{cm}^{2}$ (power: $250 \mathrm{~mW}$, diameter: $1 \mathrm{~cm}$ ) and an irradiation time of $314 \mathrm{~s}$ to achieve a final light dose of $100 \mathrm{~J} / \mathrm{cm}^{2}$.

\section{Cell viability assays}

HCT116 cells were cultured in a 96-well plate (1 $\times 10^{4}$ cells/well), and incubated with DVDMS or Photofrin at different concentrations under $37^{\circ} \mathrm{C}$ for 4 or $12 \mathrm{~h}$, the culture medium was replaced with serum free-medium, PDT treatment was proceeded and incubated for $24 \mathrm{~h}$. The cell viability was measured with CCK-8 assay kit (Dojindo Laboratories, Japan), and the data were fitted and calculated with GraphPad Prism 7.0 software to achieve the $\mathrm{IC}_{50}$.

\section{Colony formation}

HCT116 cells (400 cells/well) were seeded into 6-well plates, appropriate DVDMS or Photofrin stock solution was added to achieve designated concentration, $4 \mathrm{~h}$ later replaced PS-containing medium with fresh medium and followed by PDT treatment (the $\mathrm{CQ}$ and CQ+DVDMS groups were pretreated with CQ $(10 \mu \mathrm{M}) 1 \mathrm{~h}$ before PDT treatment). After incubating at $37^{\circ} \mathrm{C}$ for 10 days, the cells were fixed in 4\% PFA, and then stained with $0.1 \%$ crystal violet solution. The number of colonies containing more than 20 cells was counted under a microscope.

\section{DAPI staining}

For cell apoptosis analysis, the HCT116 cells $(1 \times$ $10^{6}$ cells/well) were seeded in 6-well plates. After PDT treatment, cells were incubated in $37^{\circ} \mathrm{C}$ for $4 \mathrm{~h}$, fixed in $4 \%$ PFA and incubated with DAPI. The cells were then observed under fluorescence and light microscopy (Olympus, Japan).

\section{Flow cytometry assay}

FITC Annexin V and Propidium Iodide (PI) Kit (Biolegend, USA) was used to quantify cell apoptosis according to the manufacturer's protocol. Briefly, HCT116 cells $\left(1 \times 10^{6}\right.$ cells/well $)$ were seeded in 6-well plates, after DVDMS-PDT treatment, incubated under $37^{\circ} \mathrm{C}$ for $2 \mathrm{~h}$, then trypsinized and resuspended in binding buffer, and incubated with $5 \mu \mathrm{L}$ FITC Annexin V and $10 \mu \mathrm{L}$ PI for $15 \mathrm{~min}$, and diluted with $400 \mu \mathrm{L}$ binding buffer and analyzed by flow cytometry (FACSCalibur, BD, USA). The Annexin V-FITC ${ }^{+} / \mathrm{PI}^{-}$(early period) and Annexin V-FITC ${ }^{+} / \mathrm{PI}^{+}$ (later period) cell populations were considered as the apoptotic cells.

\section{Monodansylcadaverine (MDC) staining}

The autophagic vacuoles in cells were quantified by monodansylcadaverine (MDC) stain (Leagene Biotechnology, Beijing, China) after the DVDMS-PDT treatment. $2 \mathrm{~h}$ after irradiation, the cells were collected and stained with $0.05 \mathrm{mM}$ MDC for $30 \mathrm{~min}$ at $25^{\circ} \mathrm{C}$, washed with wash buffer and resuspended with collection buffer, followed by fluorescence microscopy (excitation wavelength: $355 \mathrm{~nm}$, emission wavelength: $512 \mathrm{~nm}$; Olympus, Japan).

\section{Transmission electron microscopy (TEM) imaging}

For TEM analysis, the HCT116 cells were seeded into T75 culture flasks ( $5 \times 10^{7}$ cells/flask), and fixed in mixture contained 2\% GA and 2\% PFA for $6 \mathrm{~h}$ after PDT treatment. Then, the prefixed cells were transferred into $4 \%$ PFA for further fixation. The fixed cells were post-fixed with a $1 \% \quad \mathrm{OsO}_{4}$ buffer, dehydrated by passing through the graded alcohol steps and flat embedded in EPONTM resin. The cell samples were cut into ultra-thin sections $(100 \mathrm{~nm})$ and stained with 3\% lead citrate plus uranyl acetate and observed under an H-7650 transmission electron microscope (HITACHI, Japan).

\section{Western blotting}

Total proteins were isolated from cells with RIPA lysis buffer (Biosharp, Hefei, China) and the concentrations were determined with a BCA detection kit (Beyotime, Shanghai, China). Total protein $(50 \mu \mathrm{g})$ was separated in $8 \%$ or $10 \%$ sodium dodecyl sulfate-polyacrylamide gel electrophoresis (SDSPAGE) and transferred onto PVDF membranes (Millipore, Germany). After blocking with 5\% non-fat milk, the membranes were incubated with specific primary antibody against P62, caspase 9, caspase 3, cleaved caspase 3 and LC3 (all from Cell Signaling Technologies, USA), Bax and Bcl-2 (Abcam, UK), CD133 (Proteintech, USA), ATG7 (Novus Biologicals, USA), $\beta$-actin (Bioworld, USA) overnight at $4{ }^{\circ} \mathrm{C}$. Subsequently, samples were incubated with a HRB-conjugated anti-mouse or rabbit secondary antibody (Bioworld) at room temperature for $2 \mathrm{~h}$ and visualized by the EMD Millipore Immobilon ${ }^{\mathrm{TM}}$ Western Chemiluminescent HRP Substrate (ECL, Millipore, USA) and ECL detection system (Tanon, Shanghai, China).

\section{Animal model and treatments}

All animal experiments were performed as approved by the Institutional Animal Care and Use Committee of Second Military Medical University. Male BALB/C nude mice (16 18 g, 4 week) were supplied by Sino-British Sipper/BK Lab Animal Ltd. 
(Shanghai, China), and were fed with water and food ad libitum in an air-conditioned animal quarter under regulated temperature $\left(22 \pm 2{ }^{\circ} \mathrm{C}\right)$, relative humidity $(50 \pm 10 \%)$ and $12 \mathrm{~h}$ dark-light cycles. After seven days of acclimatization, $5 \times 10^{6}$ HCT116 cells in $100 \mu \mathrm{L}$ PBS were subcutaneously injected into the mice on the right back. Body weight and tumor sizes were measured by vernier caliper every two days till the end of the experiment and the total volume was calculated as $\mathrm{V}=\mathrm{a} \times \mathrm{b}^{2} / 2$ (a: long diameter of the tumor, b: short diameter of the tumor) [10]. When the tumors reached an average volume of $100 \mathrm{~mm}^{3}$ $\left(90-110 \mathrm{~mm}^{3}\right)$, the mice were randomly divided into different groups: (1) Control (0.9\% saline, $0.1 \mathrm{~mL}),(2)$ $5 \mathrm{mg} / \mathrm{kg} \mathrm{PF}+100 \mathrm{~J} / \mathrm{cm}^{2}$ light, (3) $10 \mathrm{mg} / \mathrm{kg} \mathrm{PF}+100$ $\mathrm{J} / \mathrm{cm}^{2}$ light, (4) $2 \mathrm{mg} / \mathrm{kg}$ DVDMS $+100 \mathrm{~J} / \mathrm{cm}^{2}$ light, and (5) $60 \mathrm{mg} / \mathrm{kg} \mathrm{CQ}+2 \mathrm{mg} / \mathrm{kg}$ DVDMS $+100 \mathrm{~J} / \mathrm{cm}^{2}$ light. CQ was given for 4 consecutive cycles by intraperitoneal injection every other day from the day before PS administration. DVDMS or PF was injected into the caudal vein, and the mice were exposed to 630 $\mathrm{nm}$ laser $24 \mathrm{~h}$ post administration [10]. All the mice were kept in dark place for 4 days after PS administration. Twenty-four hours after PDT treatment, three mice from the Control, DVDMS-PDT and CQ+DVDMS-PDT group were sacrificed, and the tumors were removed for histopathology evaluation (histological and IHC analysis). Fourteen days after the PDT treatment, all the mice were sacrificed, and the tumors were removed and weighed.

\section{Hematoxylin and eosin (HE) staining}

After fixation in 4\% PFA for at least $24 \mathrm{~h}$, tumor samples were embedded in paraffin and sectioned at a thickness of $4 \mu \mathrm{m}$. After deparaffinzation, the sections were stained with HE and observed with a light microscope (Olympus, Japan).

\section{TUNEL assay}

To identify apoptotic cells in vivo, paraffin-embedded tumor tissue sections $(4 \mu \mathrm{m})$ were stained with TUNEL in situ cell death detection kit (Roche, Switzerland) according to the instruction. Nuclei were counterstained with DAPI and showed blue under the fluorescence microscope (Olympus, Japan), and the apoptotic cells exhibited yellow-green fluorescence in the nucleus after excitation with blue light.

\section{Immunohistochemical (IHC) analysis}

For the IHC examination, the paraffin-embedded tumor tissue sections were dewaxed, rehydrated and permeabilized with $0.2 \%$ Triton X-100. To block the endogenous peroxidase, slides were immersed in 3\% $\mathrm{H}_{2} \mathrm{O}_{2}$ for 10 min. Heat-mediated antigen retrieval was performed using $10 \mathrm{mM}$ citrate buffer ( $\mathrm{pH}$ 6.0) for 10 min at $100{ }^{\circ} \mathrm{C}$ in boiling. Non-specific binding was prevented by incubated with $10 \%$ Bovine Serum Albumin (BSA) for $30 \mathrm{~min}$ at $37^{\circ} \mathrm{C}$. The sections were then incubated with primary antibodies (ki67 from abcam, LC3-II from sigma, CD133 from Proteintech) for $20 \mathrm{~h}$ at $4{ }^{\circ} \mathrm{C}$. Antibody binding was detected using anti-rabbit IgG peroxidase conjugated secondary antibodies (Bioworld technology, USA) for $30 \mathrm{~min}$ at $37^{\circ} \mathrm{C}$, and visualized using diaminobenzidine (DAB) solution (Gene Tech, Shanghai, China) conjugated with hematoxylin, and observed under light microscope (Olympus, Japan).

\section{Immunofluorescence (IF) analysis}

For IF assays, cells were cultured on glass coverslips. After the DVDMS-PDT treatment, the cells were incubated in $37{ }^{\circ} \mathrm{C}$ for $9 \mathrm{~h}$, fixed with $4 \%$ PFA and permeabilized with $0.2 \%$ Triton $\mathrm{X}-100$, and then blocked by $1 \%$ BSA. Primary antibodies (CD133, Proteintech, USA) were incubated overnight at $4{ }^{\circ} \mathrm{C}$. For IF assays, the glass coverslips were washed by PBS, incubated with second antibody (Life technologies, USA) for $1 \mathrm{~h}$ at $37^{\circ} \mathrm{C}$ and stained with DAPI. The cells were then washed and observed under fluorescence microscopy (Olympus, Japan).

\section{Statistical analysis}

Data were presented as the mean \pm standard deviation (SD). Statistical comparisons were made using one-way analysis of variance (ANOVA) and multiple comparisons between groups were performed using Turkey's test (GraphPad Prism 7.0 software), and $p<0.05\left({ }^{*}\right)$ was considered statistically significant, while $\left.p<0.01\left(^{(*)}\right), p<0.001{ }^{(* *}\right)$ and $p<$ $0.0001(* * *)$ were highly significant, respectively. The results of comparison in figures without connecting lines were compared with Control group.

\section{Results}

\section{Cellular uptake of DVDMS}

The intracellular accumulation of DVDMS was evaluated by measuring the MFI and the percentage of cells taking DVDMS by flow cytometry. As shown in Figure S1, cellular uptake of DVDMS was rapid, and more than $99 \%$ cells took DVDMS at $4 \mathrm{~h}$ after administration. However, significant increase of the MFI was observed over the subsequent hours till the end. Considering the MFI and the percentage of cells taken DVDMS, we chose $4 \mathrm{~h}$ as the time for the light exposure.

\section{DVDMS-PDT presented strong photocytotoxity and anti-proliferative effects on human colorectal cells in vitro and in vivo}

As shown in Figure 1A, DVDMS-PDT inhibited 
HCT116 cells proliferation in a dose dependent manner. To verify whether the phototoxicity was parallel between DVDMS and PF over time, $4 \mathrm{~h}$ and $12 \mathrm{~h}$ were chosen as the incubation time before PDT treatment. $24 \mathrm{~h}$ later, the data of CCK-8 was obtained and $\mathrm{IC}_{50}$ was calculated by GraphPad. Under the same condition, for both $4 \mathrm{~h}$ and $12 \mathrm{~h}$, the values $\mathrm{IC}_{50}$ of DVDMS were almost five times smaller than that of PF. Over incubation time, the phototoxicity in both group increased in the same scale. To some extent, the incubation time before illumination had little effect on the comparison of phototoxicity between DVDMS and PF. In addition, considering the cellular uptake results, we chose $4 \mathrm{~h}$ as the incubation time for the in vitro study.

Apoptosis rates of HCT116 cells in different groups were quantified (Figure $1 \mathrm{~B}$ and $1 \mathrm{C}$ ). Both DVDMS- $(100 \mathrm{ng} / \mathrm{mL}, p<0.01)$ and PF-PDT (200 $\mathrm{ng} / \mathrm{mL}, p<0.05)$ caused significant apoptosis of HCT116 cells than Control. Moreover, DVDMS-PDT $(100 \mathrm{ng} / \mathrm{mL})$ showed much higher apoptosis rate than that of PF-PDT $(200 \mathrm{ng} / \mathrm{mL}, p<0.01)$.

To evaluate the long-term anti-proliferative effects, colony formation test was performed, as shown in Figure 1D and 1E, both DVDMS- (100 $\mathrm{ng} / \mathrm{mL}, p<0.0001$ ) and PF-PDT (PF-L: $200 \mathrm{ng} / \mathrm{mL}, p<$ 0.01; PF-H: $500 \mathrm{ng} / \mathrm{mL}, p<0.0001)$ caused significant inhibition in colony formation than Control; however, no significant difference of colony counts between

DVDMS-PDT and PF-H-PDT treated cells was observed $(p>0.05)$.

\section{DVDMS-PDT triggered apoptotic cell death in human colorectal cells}

Previous studies suggested that DVDMS was accumulated in mitochondrial and induced apoptosis after exposure to light or ultrasound [18-21]. Therefore, we explored the apoptosis in HCT116 cells. First, by DAPI staining we observed classic morphological changes of apoptotic cells like condensed chromatin, nuclear fragmentation in DVDMS-PDT treated cells, and no visible nuclear changes were observed in Control, light or DVDMS alone samples (Figure 2A). Further, the apoptosis rates were quantified by flow cytometry using Annexin V-FITC/PI double staining. The data showed that DVDMS-PDT treatment $(120 \mathrm{ng} / \mathrm{mL})$ significantly induced apoptosis in HCT116 cells $(p<$ 0.0001; Figure 2B and 2C), and the apoptosis rate in light or DVDMS alone treated cells showed no difference compared with Control $(p>0.05$; Figure 2B and $2 \mathrm{C}$ ). In addition, by western blotting analysis, we observed that DVDMS-PDT upregulated the activation of caspase-9, caspase-3 and Bax in HCT116 cells (Figure 2D). These results suggested that DVDMS-PDT induced apoptotic cell death in HCT116 cells.
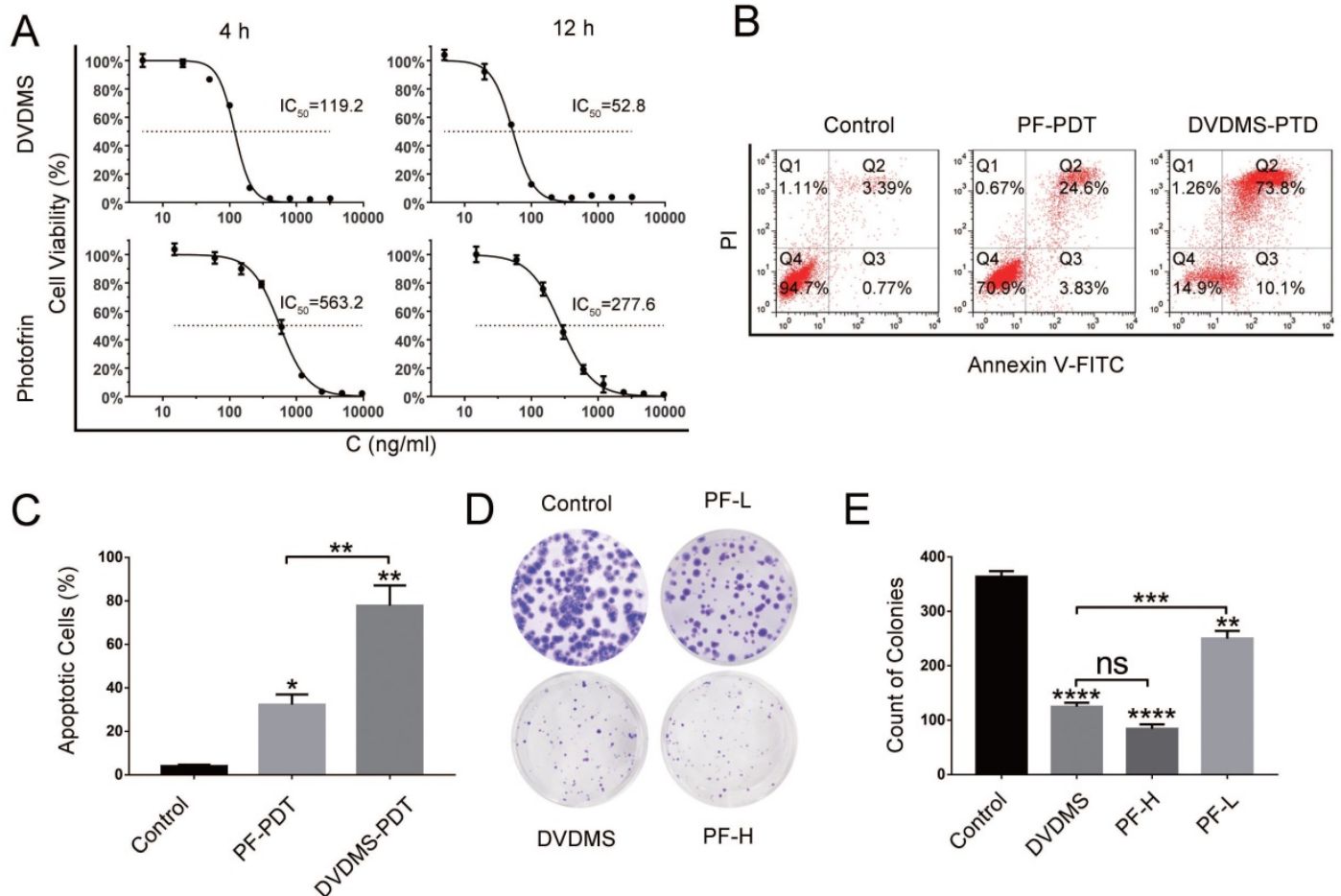

Figure 1. DVDMS-PDT presented stronger photocytotoxity and inhibiting effect on colony formation in HCT116 cells than PF-PDT. (A) The in vitro phototoxicity and $\mathrm{IC}_{50}(4$ and $12 \mathrm{~h}$ ) of DVDMS-PDT and PF-PDT in HCT116 cells determined by CCK-8. (B, C) Flow cytometry analysis of apoptosis rates in HCT116 cells treated with PF-PDT $(200 \mathrm{ng} / \mathrm{mL})$ and DVDMS-PDT (100 ng/mL). (D, E) Colony formation test in HCT116 cells treated with PF-PDT and DVDMS-PDT. The data are expressed as mean \pm SD from the results of three independent experiments, ns $p>0.05, * p<0.05, * * p<0.01, * * * p<0.001, * * * *<$ 0.0001 . 

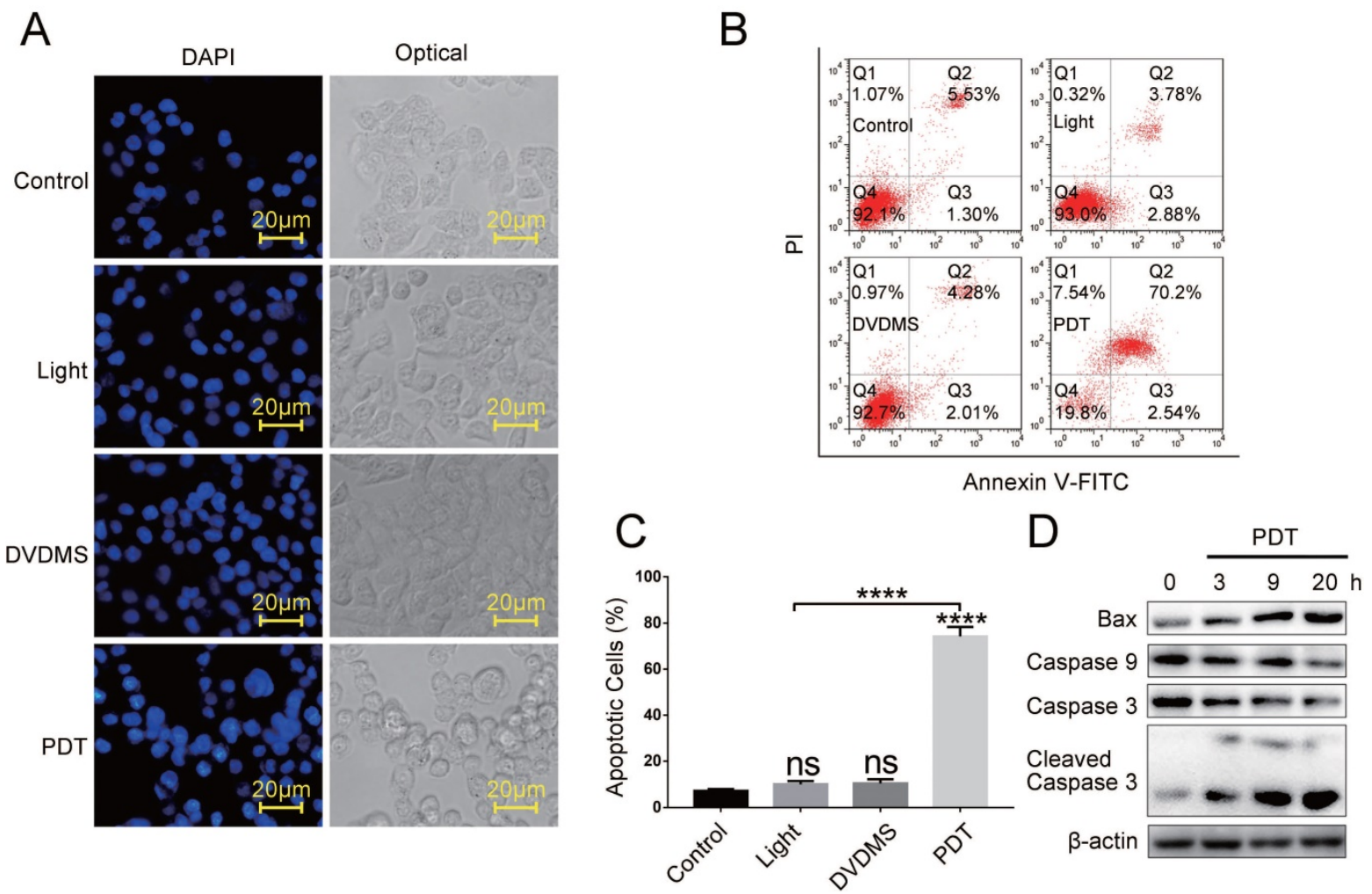

Figure 2. DVDMS-PDT induced apoptosis in HCT116 cells. (A) The morphological changes of apoptosis cells by DAPI staining ( $\times 400$ magnification). (B, C) Flow cytometry analysis of apoptosis cells in different groups. (D) Western blotting analysis of Bax, Caspase 9, Caspase 3 and Cleaved Caspase 3, $\beta$-actin was used as Control. The data are expressed as mean \pm SD from the results of three independent experiments, ns $p>0.05, * * * * p<0.0001$.

A
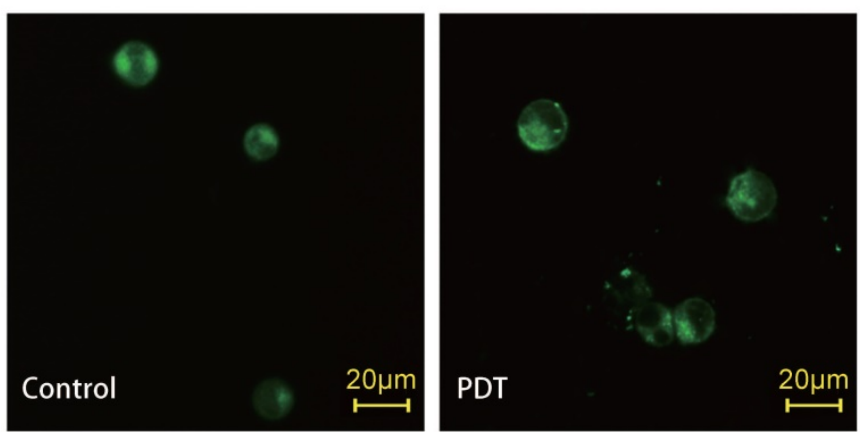

C

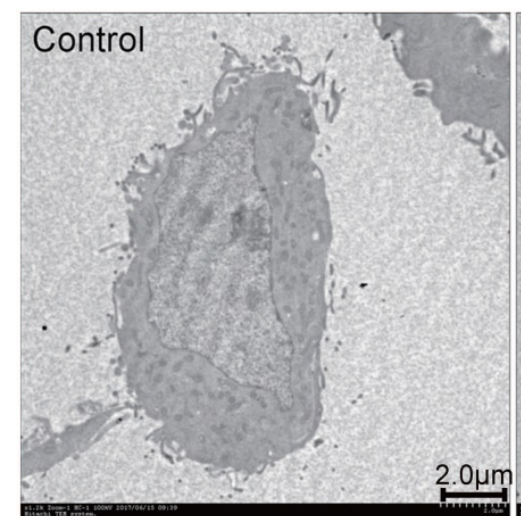

B

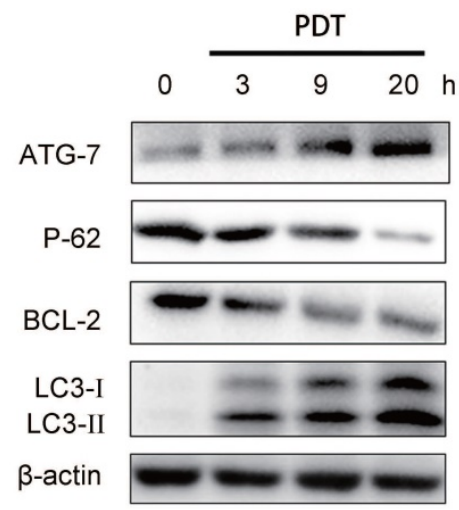

Figure 3. DVDMS-PDT induced autophagy in HCT116 cells. (A) The MDC staining ( $\times 400$ magnification) analysis of the autophagy activation in HCT116 cells. (B) Western blotting analysis of ATG7, P62, BCl-2, LC3- I and LC3- I , $\beta$-actin was used as Control. (C) Typical images of the autophagic vacuoles (indicated by the black arrow) observed under TEM. 


\section{DVDMS-PDT induced autophagy in human colorectal cells}

Numerous investigations indicated that autophagy could be induced in cancer cell lines by PDT, and some showed that autophagy plays a protective role against PDT mediated cellular damages [7, 14-16, 22, 23]. Hence, we investigated whether DVDMS-PDT can induce autophagy in HCT116 cells. First, we examined the treated cells by MDC, a specific autophagy marker aggregated in autophagosomes and autophagic lysosomes. Numerous MDC-positive spots were observed in the DVDMS-PDT treated cells compared to the cells in Control (Figure 3A). In addition, the autophagic marker LC3-II and other important autophagy-related proteins were analyzed by western blotting, which showed that the levels of LC3-II and ATG7 gradually increased at 3, 9, $20 \mathrm{~h}$ after DVDMS-PDT, while P62 and Bcl-2 gradually decreased over time (Figure 3B). These findings suggested that DVDMS-PDT could induce autophagy in HCT116 cells. To further characterize the autophagic responses upon DVDMS-PDT treatment in HCT116 cells, we performed TEM to examine the typical structures of autophagic vacuoles, and observed double membrane-bound vacuoles without ribosomes (Figure 3C) $1 \mathrm{~h}$ after PDT treatment, while the Control HCT116 cells showed normal morphology and no autophagosome was observed.

\section{DVDMS-PDT significantly inhibited tumor growth in vivo}

To verify the in vivo therapeutic efficiency of DVDMS-PDT, further experiments were performed on xenograft model in BALB/c nude mice. Representative photos of tumor-bearing mice and tumors were shown in Figure $4 \mathrm{~A}$ and $4 \mathrm{C}$. These results showed that the tumor volume $(p<0.001)$ and weight $(p<0.0001)$ were significantly reduced in PDT treated groups, and the tumor volume and weight decreased in a dose-dependent manner with the increase of PF (Figure 4D and 4E), while the body weight showed no significant difference among four groups $(p>0.05$, Figure $4 \mathrm{~B})$. The tumor volume and weight in the $2 \mathrm{mg} / \mathrm{kg}$ DVDMS group were similar to that in the $10 \mathrm{mg} / \mathrm{kg}$ PF-H group $(p>0.05)$. These results implied that DVDMS has superior antitumor efficiency than PF.

\section{Histological and IHC analysis of in vivo antitumor effects}

We then analyzed the pathophysiological changes of the tumors. By HE staining (Figure 4F), we observed that the tumor tissues from Control group displayed compact tumor cells with an intact structure. While in the PDT group, significantly increased cell apoptosis and necrosis was observed with shrunken apoptotic cells and condensed cytoplasm. In addition, to determine the effect of DVDMS-PDT on cell proliferation in vivo, IHC analysis of ki67 was performed. We observed that ki67 was highly expressed in the Control group, whereas a reduced expression was found in the DVDMS-PDT group. Increased levels of LC3-II were observed in PDT treated tumors compared to that of the tumors in Control. By IHC analysis of LC3-II, the autophagy inducing effect of DVDMS-PDT was further confirmed. Subsequently, apoptosis was measured by TUNEL and DAPI staining according to the instructions (Figure 4G), and we observed that DVDMS-PDT caused significant apoptosis in tumors. These findings confirmed the antitumor effects of DVDMS-PDT.

\section{Autophagy inhibition enhanced the apoptosis induced by DVDMS-PDT}

Subsequently, we investigated the function of autophagy induced by DVDMS-PDT in HCT116 cells. $C Q$, a specific autophagy inhibitor, which is capable of preventing autophagy by blocking autophagosome-lysosomal fusion, has been clinically used as anti-malarial and anti-inflammatory drug for a long time, whose adverse effects are well-known [24]. Nowadays, series of clinical trials were conducted and are being conducted to investigate the combined use of CQ with chemotherapy or radiotherapy for exploring the roles of autophagy inhibition, which may propose new therapeutic strategies for clinical treatments [25]. Therefore, we chose $C Q$ as the autophagy inhibition tools. First, by MDC staining we observed more active spots in CQ and PDT+CQ treated cells, suggesting successful inhibition of autophagy in corresponding cells (Figure $5 A)$. Then we observed that CQ synergistically potentiated the cytotoxicity of DVDMS-PDT treated HCT116 cells in comparison to DVDMS-PDT $(p<$ 0.001 , Figure $5 B$ and $5 C$ ). Further, the results of western blotting (cells were incubated for $9 \mathrm{~h}$ after PDT) showed that CQ enhanced DVDMS-PDTinduced expression of ATG7 and LC3-II in combined treated cells compared to cells treated with DVDMS-PDT alone. Similarly, the levels of cleaved caspase-3 were further upregulated in co-treated cells compared to the DVDMS-PDT treated cells (Figure 5D). And the colony formation assay further confirmed a superior long-term proliferationinhibiting effect of combined treatments (Figure $5 \mathrm{E}$ and $5 \mathrm{~F}$ ). Collectively, the above results suggested that autophagy may play a defensive mechanism to DVDMS-PDT treated HCT116 cells. 


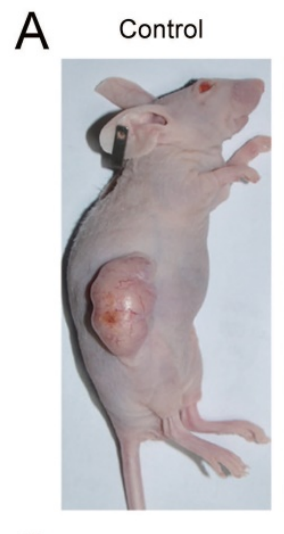

C

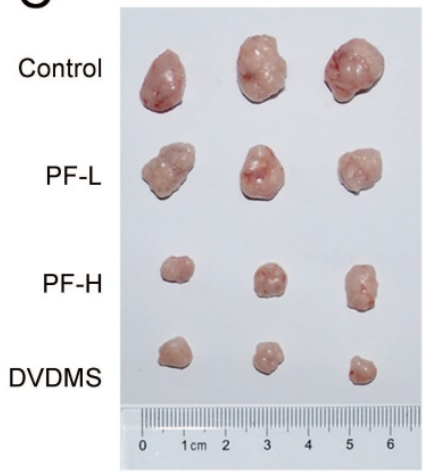

$\mathrm{F}$

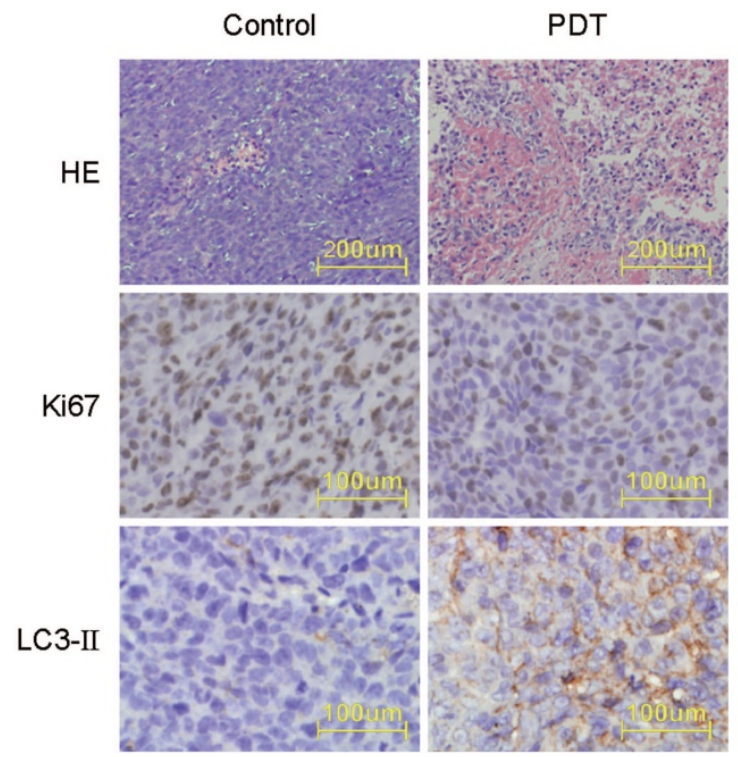

D
DVDMS
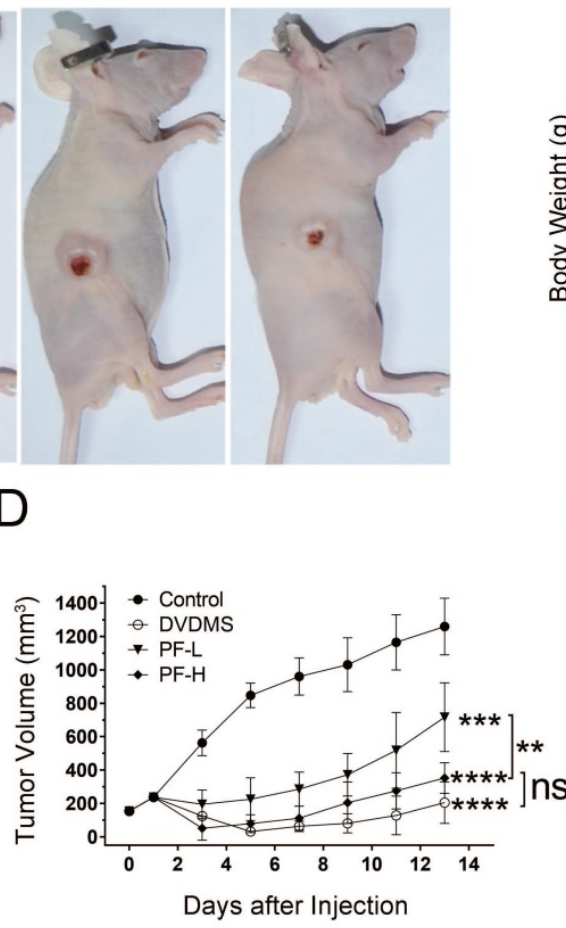

G

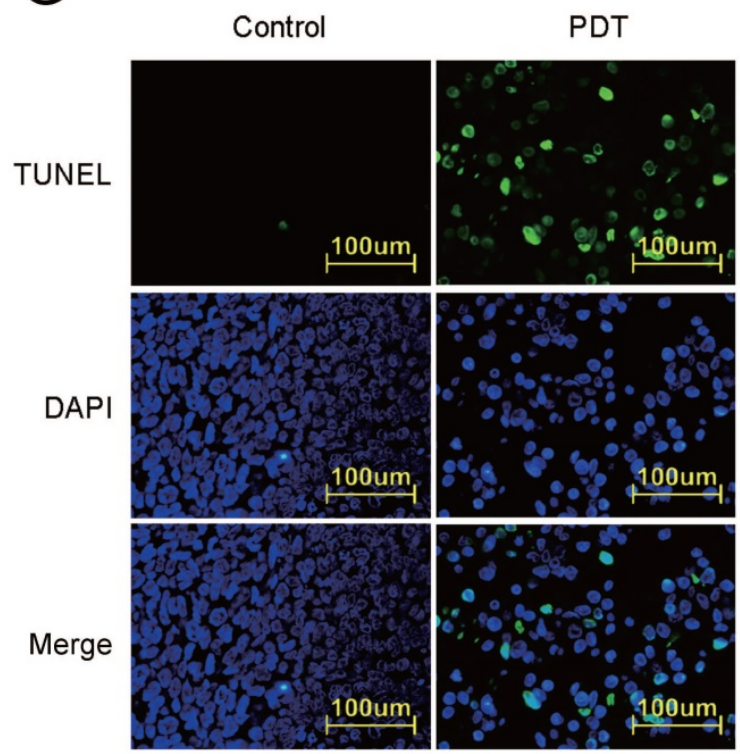

Figure 4. DVDMS-PDT exhibited better antitumor efficiency than PF-PDT in HCT116 xenograft model. Xenograft model in BALB/C nude mice implanted HCT1 16 cells were treated with Control (0.9\% saline buffer), DVDMS-PDT (DVDMS 2 mg/kg), PF-L-PDT (Photofrin 5 mg/kg) and PF-H-PDT (Photofrin 10 $\mathrm{mg} / \mathrm{kg}$ ). (A, C) Representative images of each group at the end of the xenograft model experiment. (B) Plot of body weight versus the days after injection. (D) Plot of tumor volume versus the days after injection. (E) Analysis of the tumor weight of each group at the end of the xenograft model experiment. (F) $\mathrm{HE}(\times 100$ magnification) and IHC ( $\times 400$ magnification) staining of subcutaneous implanted HCT1 16 cells from Control and DVDMS-PDT groups. (G) TUNEL and DAPI staining $(\times 400$ magnification) of subcutaneous implanted HCT1 16 cells from Control and DVDMS-PDT groups. The data are expressed as mean \pm SD $(n=5), n s p>0.05, * * p$ $<0.01, * * * p<0.001, * * * * p<0.0001$

\section{Combined treatment with DVDMS-PDT and CQ synergistically inhibited tumor growth}

To verify the in vivo therapeutic efficiency of combined treatment with DVDMS-PDT and CQ, xenograft model experiments were performed. And the representative photos of tumor-bearing mice and tumors were shown in Figure 6A and 6C. These results showed that the tumor volume and weight in the PDT+CQ group were further decreased compared to that of PDT group (tumor volume: $p<0.01$, tumor 
A
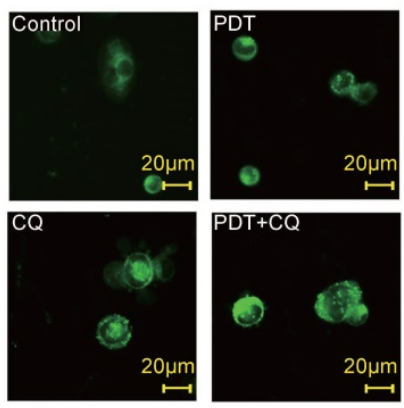

D

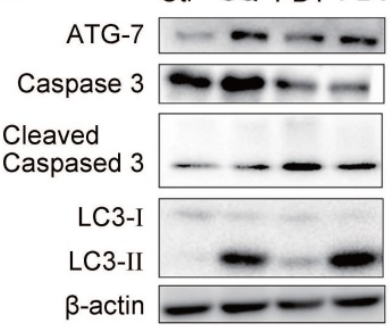

B

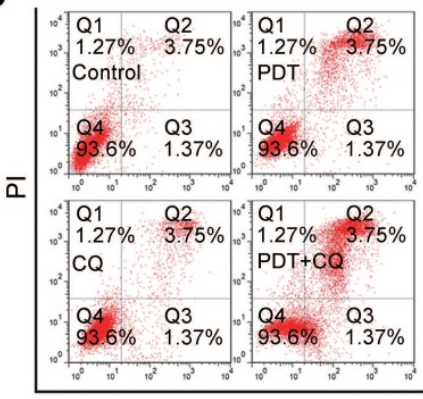

Annexin V-FITC

E

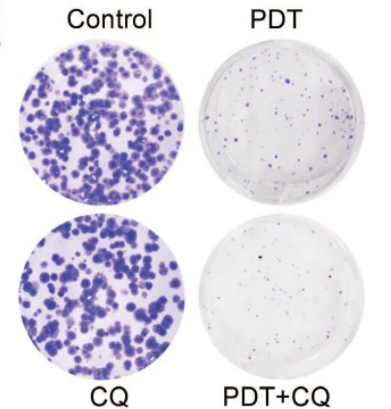

C

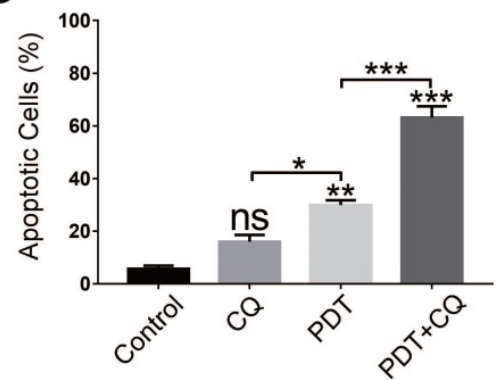

F

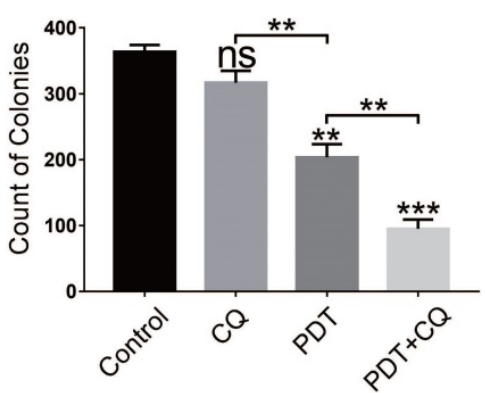

Figure 5. Inhibition of autophagy with CQ synergistically enhanced the antitumor effect of DVDMS-PDT in HCT116 cells. (A) The MDC staining ( $\times 400$ magnification) analysis of the autophagy activation in HCT116 cells of each group. Flow cytometry analysis of apoptosis rates in HCT116 cells in different groups. (D) Western blotting analysis of ATG7, Caspase 3, Cleaved Caspase 3, LC3- I and LC3- II, $\beta$-actin was used as Control. (E, F) Colony formation test in HCT116 cells in different groups. The data are expressed as mean \pm SD from the results of three independent experiments, ns $p>0.05,{ }^{*} p<0.05$, $* * p<0.01, * * * p$ $<0.001$.

weight: $p<0.05$, Figure 6D and 6E). Moreover, all the tumors in the PDT+CQ group were invisible from the fourth day post PDT treatment till the end of the in vivo experiment. Based on the results, we hypothesized that the combined treatment of autophagy inhibitor and DVDMS-PDT may reduce the recurrence of CRC. Further, we carried on western blotting, IF (cells were incubated for $9 \mathrm{~h}$ after PDT) and IHC to analyze the expression of CD133, a stem-like cell marker. And the results showed a significant decrease of CD133 expression in CQ+DVDMS-PDT group than the other two groups (Figure S2).

In addition, HE staining and IHC analysis (ki67 and LC3-II) were performed. More prominent apoptosis and necrosis, less expression of ki67 and more expression of LC3-II were observed in CQ+DVDMS-PDT group than in the other two groups. These data indicated that $\mathrm{CQ}$ inhibited autophagy in DVDMS-PDT treated HCT116 cells and promoted its antineoplastic effects (Figure 6F). And the results of TUNEL confirmed the remarkable promoting apoptosis in CQ+DVDMS-PDT group among all the three treatments (Figure 6G).

\section{Discussion}

Novel effective treatments for advanced metastatic CRC patients are urgently needed because of the severe side effects and poor prognosis of the existing clinical therapeutic approaches. PDT is a promising minimally invasive therapeutic strategy, which has been widely used in the treatments of various tumors since the 1990s, and the treatment outcomes were well-recognized [5, 9]. Photosensitizer is a key component of PDT, and PF is the only one that has been widely approved and applied in clinical cancer therapies. However, its side-effects should not be ignored, $\mathrm{PF}$ is far from the ideal one $[5,10,26]$. DVDMS is a novel PS from PF, which has been demonstrated to possess excellent properties such as high chemical purity, good water solubility and relatively short-time skin sensitivity [27]. Previous studies demonstrated that DVDMS-PDT exhibited stronger antitumor efficiency than PF-PDT in several kinds of tumor cells in vitro and in vivo [10,12, 28]. The preclinical safety study in beagle dogs demonstrated that DVDMS-PDT appeared to be a safe therapeutic modality for tumor treatments [11]. Therefore, it seems to be promising for the application of DVDMS-PDT in the therapy of CRC.

First, to assess the antitumor efficiency of DVDMS-PDT in CRC, we performed both in vitro and in vivo experiments in HCT116 cells. We used CCK-8 assay to demonstrate the phototoxicity of DVDMS. The CCK-8 assay suggested that DVDMS-PDT significantly decreased cell variability. Under the same condition, DVDMS-PDT exhibited a five times 


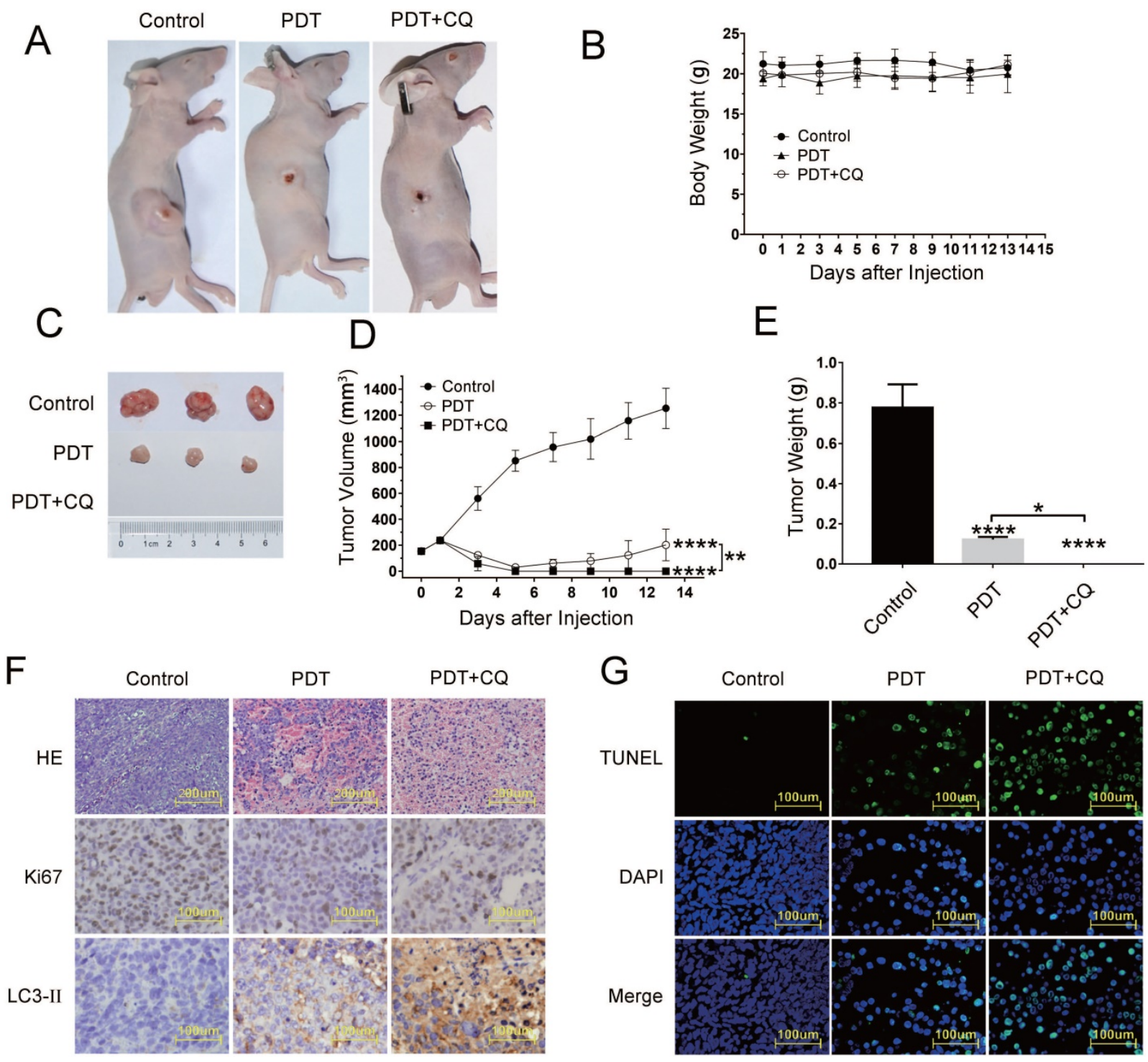

Figure 6. CQ synergistically enhanced the in vivo therapeutic efficiency of DVDMS-PDT. Xenograft model in BALB/c nude mice implanted HCT116 cells were treated with Control (0.9\% saline buffer), DVDMS-PDT (DVDMS $2 \mathrm{mg} / \mathrm{kg}$ ) or CQ+DVDMS-PDT (DVDMS $2 \mathrm{mg} / \mathrm{kg}$, CQ $60 \mathrm{mg} / \mathrm{kg}$, once every two days for five times). (A, C) Representative images of each group at the end of the xenograft model experiment. (B) Plot of body weight versus the days after injection. (D) Plot of tumor volume versus the days after injection. (E) Analysis of the tumor weight of each group at the end of the xenograft model experiment. (F) $H E(\times 100$ magnification) and IHC ( $\times 400$ magnification) staining of subcutaneous implanted HCT116 cells of each group. (G) TUNEL and DAPI ( $\times 400$ magnification) staining of subcutaneous implanted HCT116 cells from different groups. The data are expressed as mean \pm SD $(n=5), *_{p}<0.05, * * p<0.01, * * * * p<0.0001$.

higher antitumor efficiency than that of PF-PDT (Figure 1A). The results of flow cytometry and colony formation tests confirmed the antitumor effects of DVDMS-PDT. And these results suggested that DVDMS-PDT had a greater antitumor efficiency than PF-PDT under the same conditions (Figure 1). And the subsequent experiments in xenograft tumor model showed analogous results: a significant reduction in tumor volumes in DVDMS-PDT and PF-PDT groups, and the tumor size and weight in the DVDMS-PDT (2 $\mathrm{mg} / \mathrm{kg}$ ) group were similar to that of PF-PDT (10 $\mathrm{mg} / \mathrm{kg}$ ) group $(p>0.05$, Figure 4$)$. We did not observe any obvious signs of toxic side effects or changes in body weight in all the four groups, suggesting that both DVDMS and PF had no adverse effect on the growth of mice (Figure 4B).

By generating ROS, PDT could cause oxidative stress and DNA damages, which further induce pathways like apoptosis and autophagy in cancer cell lines. The PS localized in mitochondria is likely to manifest apoptosis while those localized in the plasma membrane and lysosomes cause necrosis. Apoptosis is considered the main mechanism leading to cell death in PDT. By the way, DVDMS has been shown to mainly localize in the mitochondria of several cell lines $[19,20]$, and this may render DVDMS the ability to trigger effective mitochondria-dependent apoptosis. Therefore, we examined the apoptosis inducing effects of DVDMS-PDT in HCT116 cells by morphological changes and Annexin V-FITC/PI, 
which was further confirmed by the results of western blotting with increased ratio of the Bax/Bcl-2 and activated caspase-3, and decreased levels of the caspase-9 and caspase-3 (Figure 2). Most likely, HCT116 cells were significantly inhibited by DVDMS-PDT-induced apoptotic cell death. Autophagy is found to be the main process of cell death where apoptotic mechanism is absent, and it may play a prosurvival role in apoptosis competent cells and a prodeath role in deficient ones [29]. Therefore, we examined one of the markers of autophagy, autophagic vacuoles, whose accumulation were observed by MDC staining plus fluorescence microscopy and TEM, which was further confirmed by dramatically changes of typical autophagy-related proteins (upregulation: LC3-II, ATG7; downregulation: Bcl-2, P62; Figure 3). These data suggested that DVDMS-PDT triggered apoptosis and autophagy in HCT116 cells. And the results of HE, IHC and TUNEL tests (Figure $4 \mathrm{~F}$ and $4 \mathrm{G}$ ) of removed tumors in different groups confirmed the antitumor effects of DVDMS-PDT and preliminarily disclose the mechanism, which may be related to apoptosis, autophagy and proliferation inhibition.

The mechanisms regulating autophagy cell death or survival are still elusive. Depending on the type of PS and ROS, presence of apoptotic mechanism and extent of photodamage, autophagy may be cytoprotective or cytotoxic [30]. To determine the role of autophagy in DVDMS-PDT for CRC, we chose CQ, a late-stage autophagy inhibitor. Subsequently, we observed that $\mathrm{CQ}$ synergistically enhanced the DVDMS-PDT induced cell apoptosis (Figure 5B and 5C) and inhibition of colony formation (Figure 5E and 5F) compared to cells treated with DVDMS-PDT alone by flow cytometry analysis and colony formation test. As expected, the accumulation of autophagic vacuoles and increased levels of LC3-II and ATG7 implied successfully blocking of autophagy by CQ. Meanwhile, we observed upregulation of activated caspase-3, a typical marker of cell apoptosis (Figure 5D).

More importantly, similar results were achieved in the xenograft tumor model. As expected, the body weight of mice in all three groups showed no significant difference during the experiment (Figure $6 \mathrm{~B}$ and $6 \mathrm{C}$ ), implying that the therapeutic modality and dosage of administration are relatively safe. Interestingly, the tumors in DVDMS-PDT and CQ+DVDMS-PDT were hardly detectable at the fifth day post the PDT treatment, and over the following eight days the tumors in DVDMS-PDT group were significantly smaller than Control group but could be detected, while in CQ concomitant treated group the tumors were hardly detectable till the end (Figure
6D). Finally, by HE, IHC and TUNEL tests, the results in different groups (Control, DVDMS, DVDMS+CQ) confirmed that autophagy was successfully blocked by $C Q$, which synergistically enhanced the efficiency of tumor inhibition (Figure 6F and 6G). Based on these results, we hypothesized that the combined treatment with autophagy inhibitor and DVDMS-PDT may reduce the recurrence of CRC. Recently, a study suggested that autophagy promoted resistance to PDT-induced apoptosis selectively in colorectal cancer stem-like cells [8]. In the previous studies, cancer stem cell-like property has been observed in the majority of HCT116 cells, and CD133 was considered as a theoretical cancer stem-like cell marker for colorectal cancer, HCT116 cells with CD133 overexpression have been demonstrated with enhanced tumor-initiating ability and tumor relapse probability [31-33]. Therefore, we detected CD133 in vitro (western blotting, IF) and in vivo (IHC). The results implied that the CQ may synergistically sensitize stem-like cells in HCT116 cell line to DVDMS-PDT, and eventually results in decrease of tumor recurrence (Figure S2), and the relevant conclusion needs further study.

In conclusion, our study demonstrated that DVDMS-PDT is more effective in inhibiting HCT116 cells than PF-PDT, and that both apoptosis and autophagy are induced in DVDMS-PDT treated HCT116 cells. Moreover, inhibition of autophagy in DVDMS-PDT treated HCT116 cells significantly potentates the antitumor efficiency. Altogether, DVDMS might be a promising PS, and the combined use autophagy inhibitor with DVDMS-PDT appears to be a feasible therapeutic regimen for CRC treatment.

\section{Supplementary Material}

Supplementary figures and tables.

http://www.ijbs.com/v15p0012s1.pdf

\section{Acknowledgments}

This research was supported by the National Key Research and Development Program of China (2018YFA0107503), the National Natural Science Foundation of China (No.81472846) and the Fundamental Research Funds for the Central Universities (GK201502009). The authors thank department of biophysics (College of Basic Medical Sciences, Second Military Medical University) for performing the TEM imaging. The authors thank Qinglong Hi-tech Co., Ltd for providing the novel sensitizer DVDMS.

\section{Authors' contributions}

Bing Zhu and Shanxin Li initiated, detailed and 
carried out the experiments. Lei Yu, Bing Zhu and Shanxin Li prepared the manuscripts and figures. Lei $\mathrm{Yu}$ and Naping Zhao helped perform the data analysis. Xiaojuan Hou, Dandan Sheng and Yechen $\mathrm{Wu}$ provided experimental suggestion. Wei $\mathrm{Hu}$, Zhipeng Han, Xiaojuan Hou and Jing Hou contributed to the preparation and editing of the final version of the manuscript and figures. Lixin Wei and Li Zhang initiated and designed the experiments.

\section{Competing Interests}

The authors have declared that no competing interest exists.

\section{References}

1. Chen W, Zheng R, Baade PD, Zhang S, Zeng H, Bray F, et al. Cancer statistics in China, 2015. CA Cancer J Clin. 2016; 66: 115-32.

2. Siegel RL, Miller KD, Jemal A. Cancer statistics, 2016. CA Cancer J Clin. 2016; 66: 7-30

3. Siegel RL, Miller KD, Fedewa SA, Ahnen DJ, Meester RGS, Barzi A, et al. Colorectal cancer statistics, 2017. CA Cancer J Clin. 2017; 67: 177-93.

4. Mulsow J, Merkel S, Agaimy A, Hohenberger W. Outcomes following surgery for colorectal cancer with synchronous peritoneal metastases. Br J Surg. 2011; 98: 1785-91.

5. van Straten D, Mashayekhi V, de Bruijn HS, Oliveira S, Robinson DI. Oncologic Photodynamic Therapy: Basic Principles, Current Clinical Status and Future Directions. Cancers. 2017; 9: doi: 10.3390/cancers9020019.

6. Nakamura T, Fukui H, Ishii Y, Ejiri K, Ejiri M. Photodynamic therapy with polypectomy for rectal cancer. Gastrointest Endosc. 2003; 57: 266-9.

7. Xiong L, Liu Z, Ouyang G, Lin L, Huang H, Kang H, et al. Autophagy inhibition enhances photocytotoxicity of Photosan-II in human colorectal cancer cells. Oncotarget. 2017; 8: 6419-32.

8. Wei MF, Chen MW, Chen KC, Lou PJ, Lin SY, Hung SC, et al. Autophagy promotes resistance to photodynamic therapy-induced apoptosis selectively in colorectal cancer stem-like cells. Autophagy. 2014; 10: 1179-92.

9. Benov L. Photodynamic therapy: current status and future directions. Med Princ Pract. 2015; 24 Suppl 1: 14-28.

10. Wang X, Hu J, Wang P, Zhang S, Liu Y, Xiong W, et al. Analysis of the in vivo and in vitro effects of photodynamic therapy on breast cancer by using a sensitizer, sinoporphyrin sodium. Theranostics. 2015; 5: 772-86.

11. Lin N, Li C, Wang Z, Zhang J, Ye X, Gao W, et al. A safety study of a novel photosensitizer, sinoporphyrin sodium, for photodynamic therapy in Beagle dogs. Photochem Photobiol Sci. 2015; 14: 815-32.

12. Shi R, Li C, Jiang Z, Li W, Wang A, Wei J. Preclinical Study of Antineoplastic Sinoporphyrin Sodium-PDT via In vitro and In vivo Models. Molecules. 2017; 22: doi: $10.3390 /$ molecules22010112.

13. Kimmelman AC. The dynamic nature of autophagy in cancer. Genes Dev. 2011; 25: 1999-2010.

14. Andrzejak M, Price M, Kessel DH. Apoptotic and autophagic responses to photodynamic therapy in 1c1c7 murine hepatoma cells. Autophagy. 2011; 7: 979-84.

15. Xue LY, Chiu SM, Oleinick NL. Atg7 deficiency increases resistance of MCF-7 human breast cancer cells to photodynamic therapy. Autophagy. 2010; 6: 248-55.

16. Kessel D, Arroyo AS. Apoptotic and autophagic responses to Bcl-2 inhibition and photodamage. Photochem Photobiol Sci. 2007; 6: 1290-5.

17. Kessel D, Reiners JJ Jr. Apoptosis and autophagy after mitochondrial or endoplasmic reticulum photodamage. Photochem Photobiol. 2007; 83: 1024-8.

18. Xiong $\mathrm{W}$, Wang $\mathrm{X}, \mathrm{Hu} \mathrm{J}$, Liu $\mathrm{Y}$, Liu $\mathrm{O}$, Wang $\mathrm{P}$. Comparative study of two kinds of repeated photodynamic therapy strategies in breast cancer by using a sensitizer, sinoporphyrin sodium. J Photochem Photobiol B. 2016; 160: 299-305.

19. Wu L, Wang X, Liu Q, Wingnang Leung A, Wang P, Xu C. Sinoporphyrin sodium mediated photodynamic therapy inhibits the migration associated with collapse of F-actin filaments cytoskeleton in MDA-MB-231 cells. Photodiagnosis Photodyn Ther. 2016; 13: 58-65.

20. Wang $\mathrm{H}$, Wang $X$, Zhang S, Wang $\mathrm{P}$, Zhang K, Liu Q. Sinoporphyrin sodium, a novel sensitizer, triggers mitochondrial-dependent apoptosis in ECA-109 cells via production of reactive oxygen species. Int J Nanomedicine. 2014; 9: 3077-90.

21. Wang H, Wang P, Li L, Zhang K, Wang X, Liu Q. Microbubbles Enhance the Antitumor Effects of Sinoporphyrin Sodium Mediated Sonodynamic Therapy both In vitro and In vivo. Int J Biol Sci. 2015; 11: 1401-9.

22. Huang Q, Ou YS, Tao Y, Yin H, Tu PH. Apoptosis and autophagy induced by pyropheophorbide-alpha methyl ester-mediated photodynamic therapy in human osteosarcoma MG-63 cells. Apoptosis. 2016; 21: 749-60.
23. Dewaele M, Martinet W, Rubio N, Verfaillie T, de Witte PA, Piette J, et al. Autophagy pathways activated in response to PDT contribute to cell resistance against ROS damage. J Cell Mol Med. 2011; 15: 1402-14.

24. Ch'ng JH, Lee YQ, Gun SY, Chia WN, Chang ZW, Wong LK, et al. Validation of a chloroquine-induced cell death mechanism for clinical use against malaria. Cell Death Dis. 2014; 5: e1305. doi: 10.1038/cddis.2014.265.

25. Solomon VR, Lee H. Chloroquine and its analogs: a new promise of an old drug for effective and safe cancer therapies. Eur J Pharmacol. 2009; 625: 220-33.

26. Yano S, Hirohara S, Obata M, Hagiya Y, Ogura S, Ikeda A, et al. Current states and future views in photodynamic therapy. J Photochem Photobiol C. 2011; 12: 46-67.

27. Fang Q, Yang D. A porphyrin dimer sodium salt combined with the ether bond and its manufacturing method. [P]. 2012; ZL200910179116.5. China.

28. Jiang $\mathrm{Z}$, Shi $\mathrm{R}$, Li C, Wang A. Inhibitory effects of DVDMS-2-based-photodynamic therapy on the growth of tumor in vitro and in vivo. Carcinogenesis,Teratogenesis \& Mutagenesis. 2013; 25: 163-7.

29. Reiners JJ Jr., Agostinis P, Berg K, Oleinick NL, Kessel D. Assessing autophagy in the context of photodynamic therapy. Autophagy. 2010; 6: 7-18.

30. Chilakamarthi U, Giribabu L. Photodynamic Therapy: Past, Present and Future. Chem Rec. 2017; 17: 775-802.

31. Chen K, Jiang H, Chen J, Pei L. Stem cell-like property of colon cancer cell line HCT116. Med J Chin PLA. 2009; 34: 1292-6.

32. Chen K, Pan F, Jiang H, Chen J, Pei L, Xie F, et al. Highly enriched CD133(+)CD44(+) stem-like cells with CD133(+)CD44(high) metastatic subset in HCT116 colon cancer cells. Clin Exp Metastasis. 2011; 28: 751-63.

33. Ning S, Lee S, Wei M, Peng C, Lin S, Tsai M, et al. Targeting Colorectal Cancer Stem-Like Cells with Anti-CD133 Antibody-Conjugated SN-38 Nanoparticles. ACS Appl Mater Interfaces. 2016; 8: 17793-804. 